\title{
Susan O'Regan, Music and Society in Cork, 1700-1900
}

\section{Yves Defrance}

\section{(2) OpenEdition}

\section{Journals}

\section{Édition électronique}

URL : https://journals.openedition.org/etudesirlandaises/12147

DOI : 10.4000/etudesirlandaises. 12147

ISSN : 2259-8863

\section{Éditeur}

Presses universitaires de Caen

\section{Édition imprimée}

Date de publication : 30 décembre 2021

Pagination : 166-171

ISBN : 978-2-84133-157-0

ISSN : 0183-973X

\section{Référence électronique}

Yves Defrance, «Susan O'Regan, Music and Society in Cork, 1700-1900», Études irlandaises [En ligne], 46-2 | 2021, mis en ligne le 17 décembre 2021, consulté le 10 novembre 2022. URL : http://

journals.openedition.org/etudesirlandaises/12147; DOI : https://doi.org/10.4000/etudesirlandaises. 12147

\section{(c) (i) (2)(2)}

Creative Commons - Attribution - Pas d'Utilisation Commerciale - Partage dans les Mêmes Conditions 4.0 International - CC BY-NC-SA 4.0

https://creativecommons.org/licenses/by-nc-sa/4.0/ 
continues to make the same stereotype that was made when he was deselected in 1959. Outside Northern Ireland, the stereotype of unionism as reactionary and ultra-conservative remains and although many continue to show there are different facets to unionism, their voices still struggle to be heard. The real value of this book is not as critique or biography but as a challenge to the deafness of unionism to its own history.

David SHAw

\section{Susan O'Regan, Music and Society in Cork, 1700-1900, Cork, Cork Uni- versity Press, 2018, 364 p.}

Comme l'indique clairement son titre, cette monographie traite de la vie musicale à Cork aux XVIII ${ }^{e}$ et XIX ${ }^{e}$ siècles, périodes sur lesquelles les études de ce type sont quasi inexistantes. Pour mener à bien son projet, l'auteure a puisé à diverses sources historiques. L'imposante bibliographie, exclusivement en langue anglaise, comprend, certes, un très grand nombre d'ouvrages. Elle est surtout enrichie d'une multitude de journaux, périodiques, affiches et programmes de théâtres ou de concerts, complétés d'une collection de manuscrits conservés en divers lieux de la ville comme les archives cléricales locales, celles de la cathédrale Saint-Finbarr ou de sociétés savantes, académies ou établissements d'enseignement musical. Un appareil de près de 1200 notes est renvoyé par chapitre en fin de volume ce qui favorise une première lecture fluide mais demande, comme toujours dans ce mode de présentation, un va-et-vient entre les pages dès que l'on cherche à prendre connaissance de l'origine d'une référence. À cela s'ajoutent d'utiles index et tables diverses: illustrations, abréviations, termes musicaux et même un rappel des valeurs monétaires (livre, shilling, guinée). Les illustrations, on comprend aisément pourquoi, sont rares au XVIII siècle et apparaissent principalement dans les quatre derniers chapitres correspondant à la période la plus récente. Enfin, un plan du centre-ville de Cork permet de localiser seize principaux lieux de concerts et de pratique publique musicale dont une bonne part reste observable de nos jours.

Nous avons là un ouvrage à la documentation solide présenté en dix chapitres selon un ordre chronologique: la deuxième ville d'Irlande/les concerts publics au XVIII siècle / musique et Église jusqu'en 1820 / musique lyrique jusqu'en 1840 / musique de charité et de divertissement/ citadins talentueux, mobilité et opportunité, 1820-1840 / explosion culturelle, diversité de la scène musicale, 18301840 / la propagation de la pratique amateure, 1840-1870 / musique et catholicisme, 1800-1900 / un avenir glorieux.

Ce découpage à la fois thématique et historique correspond à l'histoire même de la cité portuaire qui jouit d'une position stratégique propre à attirer armateurs et négociants. Juxtaposition des Églises anglicane et catholique romaine, coexistence d'Irlandais et d'Anglais, bilinguisme et opposition de groupes sociaux qui se doivent 
de cohabiter. De sorte que la vie musicale à Cork reflète inévitablement la société quelque peu bigarrée qui s'y est épanouie. Comme tout grand port, Cork est ouvert vers l'extérieur, favorisant ainsi l'influence de modèles culturels urbains venus d'ailleurs. Ainsi, les représentants de la bonne société adoptent les mœurs et habitudes des capitales européennes et ne sont pas en reste quant aux modes vestimentaires et de distractions. Il devient alors logique d'y rencontrer la pratique de musiques classiques continentales comme le baroque galant italien, puis le style italianisant de Rossini, tout autant que l'opéra-ballade anglais ${ }^{3}$, les glees anglais ${ }^{4}$ ou le style romantique allemand. Cette tendance s'amplifie au XIX siècle au point qu'il faille agrandir les salles de spectacle pour accueillir des artistes européens en tournée. La diversité des programmes en témoigne, de même que la tendance à favoriser la virtuosité, effet qui profite à la pratique locale professionnelle mais aussi amateure.

Susan O'Regan n'en est pas à son coup d'essai. Elle connaît bien son sujet pour avoir soutenu une thèse de doctorat en 2008 au Cork Institute of Technology sous l'intitulé Public Concerts in the Musical Life in Cork 1754-1840. À présent la problématique englobe deux siècles. Elle s'étend aux périodes antérieures et postérieures à celle retenue dans le cadre du travail universitaire qui, lui, sert de socle central au texte. À l'intérieur de ce large panorama historique certains événements politiques semblent agir comme catalyseurs de prises de conscience aux répercussions importantes dans la vie culturelle d'une ville. Suite à l'Acte d'Union (1801), qui établit pour plus d'un siècle un Royaume-Uni de Grande-Bretagne et d'Irlande, on assiste à l'éveil général de l'identité irlandaise dans la revalorisation de son passé. L'orgueil national renaissant se manifeste assez nettement dans la production de chansons, en particulier celles de Thomas Moore, ou dans l'expansion du jeu de la harpe irlandaise sur la scène publique ${ }^{5}$. Cette réalité nouvelle a pour effet de combiner des savoirs relevant des musiques écrites continentales (fine art music) et d'autres de transmission orale liés aux diverses traditions de l'île (folk music). Bel exemple de rencontre entre le populaire et le savant pas toujours aisés à concilier en Europe.

3. Le ballad opera est une forme d'opéra comique proprement anglais comprenant des chants entrecoupés de dialogues. Le plus célèbre et en même temps précurseur du genre fut The Beggar's Opera (1728) avec un texte de Johann Christoph Gay et une adaptation musicale de John Pepusch. Il s'en écrivit encore au XIX ${ }^{\mathrm{e}}$ siècle.

4. Le glee est un genre vocal polyphonique anglais souvent pratiqué dans des clubs qui lui sont dédiés. En vogue aux XVIII ${ }^{\mathrm{e}}$ et XIX ${ }^{\mathrm{e}}$ siècles, il est d'ordinaire chanté à trois voix ou plus, a cappella, i.e. sans accompagnement instrumental, dans une écriture plus homophonique que contrapunctique. Les glees, considérés comme héritiers des madrigaux anglais du $\mathrm{XVI}^{\mathrm{e}}$ siècle, remplissent une fonction sociale dans les milieux plutôt aisés et s'adressent principalement à d'habiles amateurs qui en firent un marqueur de distinction. De plus, ils permirent de maintenir une tradition proprement anglaise à une époque où bon nombre de compositeurs insulaires adoptaient les styles musicaux venus du continent suivant les modèles de Haendel, Haydn et plusieurs de leurs contemporains, ainsi qu'un groupe de disciples de Beethoven. La plupart des glees nous vient de compositeurs anglais ayant vécu entre 1750 et 1825 .

5. Rappelons que le coup d'envoi de la renaissance de la harpe diatonique irlandaise et de la prise de conscience de l'importance de la musique dans la construction de l'identité irlandaise semble avoir été donné suite au succès du concours de harpe organisé à Belfast à une date très symbolique, le 14 juillet 1790 . 
Les programmes des concerts et parades des orchestres militaires examinés dans ce livre montrent aussi l'étendue et la variété des répertoires interprétés en public, souvent à titre gracieux et en plein air. Ces modèles popularisèrent des esthétiques et œuvres nouvelles. Ils incitèrent sans doute les spectateurs à se rendre eux-mêmes dans les sociétés musicales qui se multiplient au XIX ${ }^{\mathrm{e}}$ siècle et offrent, à leur tour, des programmes de concerts ouverts aux nouveaux publics. Des troupes de musiciens et chanteurs réputés commencèrent à venir plus régulièrement de Dublin, de Londres et parfois du continent. Les tournées de compositeurs et interprètes virtuoses comme le violoniste italien Niccolò Paganini (1782-1840) ou le pianiste hongrois Franz Liszt restèrent gravées dans les mémoires depuis leur passage à Cork respectivement en septembre 1831 et fin décembre 1840. Comme partout, la presse s'en fit l'écho. Son rôle est ici bien analysé tant dans les comptes rendus de concerts que dans la publicité faite en amont sur de tels événements musicaux. Il est intéressant de relever combien la presse locale participe de ce mouvement d'incitation à se rendre au concert. Elle attire foule aux concerts, en donne des comptes rendus et annonce de nouvelles manifestations futures. Déterminante dans le dynamisme des activités des nombreuses sociétés musicales de la ville, elle témoigne des querelles et rivalités favorisant de la sorte une réelle émulation collective.

Une bonne partie de l'intérêt de ce livre réside dans le fait que Susan O'Regan ne se contente pas d'énumérer des faits historiques. Elle élargit son angle d'observation à la société citadine qui les produit. En effet, derrière une somme d'informations factuelles, qui vont jusqu'à donner les noms propres des musiciens d'orchestre, se révèlent quantité de jeux de pouvoir et d'équilibre entre les diverses couches de la société avec leurs réseaux, leurs tendances esthétiques ou l'affirmation de leur position sociale à travers leurs choix. Quand certaines manifestations musicales de grande ampleur, telle l'inauguration d'une salle de spectacle, tendent à rassembler, d'autres initiatives divisent et laissent transparaître d'inévitables clivages sociaux et politiques. Cependant, le développement des sociétés musicales vient contrecarrer les habitudes anciennes de la haute société. Le chapitre huit, particulièrement soigné, est entièrement consacré à l'explosion des pratiques amateures. Il y est démontré que le tournant semble avoir été pris après 1840, date à partir de laquelle s'affirme une réelle volonté d'éducation musicale populaire. Comme le rappelle l'auteure, «malgré les tensions politiques et sectaires, les sociétés de chant choral se distinguent comme élément clé de la vie sociale de la classe moyenne à la fin du XIX ${ }^{\mathrm{e}}$ siècle ${ }^{6}$. Petit à petit, cette classe émergente accède à l'éducation musicale, soutenue par des œuvres charitables et organismes qui lui sont dédiés. Une telle approche historique et sociale permet en effet de mieux apprécier le poids des institutions comme l'Église, l'État, les collectivités locales ou, dans une moindre mesure, l'Armée, dans le maintien d'un niveau élevé de pratique musicale. La cathédrale Saint-Finbarr (achevée en 1889)

6. «Despite political and sectarian tensions, choral societies emerged as a key element of middle-class sociability during the late nineteenth century» ( $4^{\mathrm{e}}$ de couverture; ma traduction). 
sut entretenir un noyau de musiciens professionnels au rayonnement suffisant pour dispenser un enseignement musical de qualité. Les chorales amateures s'en inspirent et, à partir des années 1840, ne se contentent plus d'intervenir dans le seul cadre des offices religieux. Ce phénomène, observé dans bon nombre de régions d'Europe continentale notamment les pays scandinaves, baltes, magyars ou slaves, participe d'un mouvement romantique général d'éducation populaire mais aussi de réveil des «petites nations» et d'écoles musicales «nationales». On pense aux cinq Russes mais également aux Grieg, Nielsen, Kuhlau, Smetana, Dvorak, Sibelius, Bartók ou Kodaly. Un chapitre sur la création musicale à Cork aurait utilement complété ce panorama. Chacun garde en mémoire le destin remarquable du grand compositeur dublinois John Field (1782-1837), considéré comme l'inventeur du nocturne pour piano, forme popularisée par Chopin, puis Balakirev ou Debussy ${ }^{7}$. Celui de Philip Cogan (1750-1833) né à Cork est ici passé sous silence. Cogan, lui-même enfant de chœur éduqué à Cork, puis chantre (vicar choral) à Saint-Finbarr, devint organiste à la cathédrale Saint-Patrick de Dublin. Il fut cofondateur de la Irish Musical Fund Society et laissa une œuvre non négligeable d'opéras et de pièces pour piano avec notamment un concerto pour piano (1790) dans lequel il cite la chanson populaire française "Malbrough s'en va-t-en guerre». Mais le plus surprenant est que, dans les périodes qui précèdent l'avènement d'Aloys Fleischmann (1910-1992), peu ou pas de compositeurs nés à Cork ou ayant écrit leur œuvre dans cette ville ne soient mentionnés dans ce travail ${ }^{8}$.

Susan O'Regan montre pourtant bien comment, durant la seconde partie du XIX ${ }^{\mathrm{e}}$ siècle, différents faits concomitants placent la musique comme élément déterminant dans l'essor de la vie sociale à Cork. La ville aux «nouvelles églises catholiques» se dote bien entendu de structures musicales religieuses. Mais l'offre culturelle ne suffit plus. Il faut désormais ouvrir une école de musique municipale accessible au plus grand nombre. Les pratiques amateures prennent alors une ampleur inédite. Conséquence d'une sorte de démocratisation de l'enseignement musical et de son développement, une partie de la population, jusque-là principalement versée dans les musiques populaires et traditionnelles, commence à faire sienne une autre forme de sociabilité. À partager goût et pratique de la musique écrite occidentale cette part croissante de la population citadine en assimile aussi les codes sociaux. De sorte qu'un nouveau public se dispose à sortir d'une espèce de vase clos, à se déplacer et même à payer sa place pour goûter aux plaisirs des concerts autrefois réservés à une élite sociale irlandaise et anglaise. Une nouvelle demande de spectacles de qualité (high class music) se fait sentir et pousse les élus à faire construire plusieurs salles de spectacle, notamment le superbe Cork Opera House, inauguré en 1855. Dès lors, les organisateurs de programmes musicaux

7. Voir les enregistrements récents de nocturnes faits par le talentueux pianiste David Quigley.

8. Fils d'un couple de musiciens (sa mère enseignait le piano à Cork où elle était née) et petit-fils d'Allemands établis à Cork, Fleischmann s'activa à revaloriser la musique classique irlandaise, notamment à Cork. On lui doit un ouvrage de référence: Music in Ireland: A Symposium, Cork, Cork University Press, 1952. 
multiplient les invitations de troupes de chanteurs et musiciens en tournée en Irlande et en Grande-Bretagne. Dans un esprit à la fois philanthropique et mercantile ils cherchent à jouer à guichets fermés en proposant des billets à bas prix. Cork veut rayonner dans tout le sud de l'île au shamrock et s'inspire de ce qui se fait ailleurs, notamment à Dublin, Londres et Paris. On le sait, les Irlandais s'intéressent de longue date à la Révolution française et restent à l'écoute des idées, manières et modes en tout genre qui animent les régimes lui succédant sur le continent. Relevons au passage l'importance de compositeurs et chefs d'orchestre français comme Louis Julien, établi à Londres et reprenant à son compte le «concert promenade» mis au point par son collègue parisien Philippe Musard. Le concept et son application avaient jailli dès la fin des années 1830 à Paris afin de rendre la musique accessible aux classes laborieuses. De là l'expression anglaise de «shilling audiences» qui traduit bien la volonté de conquête de spectateurs. Louis Julien vint d'ailleurs jouer à Cork en 1855. Ces décisions majeures de la part des responsables culturels de Cork tendent à décloisonner la vie musicale locale. Elles participent aussi à l'évolution de la vie sociale et, par ricochet, à l'attractivité de la cité en pleine expansion. On voit là combien une politique culturelle municipale, en l'occurrence musicale, peut agir tant sur le comportement d'une population citadine en interne que sur l'image qu'elle donne d'elle-même à l'extérieur.

Tout ceci n'est pas bien nouveau en soi mais Susan O'Regan laisse le lecteur en mesurer le poids grâce aux nombreux témoignages et anecdotes qui en agrémentent le récit. On aurait souhaité en connaître encore davantage et surtout bénéficier d'une synthèse véritablement sociologique. Je regrette en particulier l'absence de références majeures comme les travaux de Norbert Elias puis ceux de Pierre Bourdieu sur les rouages de la différenciation sélective à travers les pratiques culturelles des diverses composantes d'une société donnée ${ }^{9}$. Il apparaît que cette vie musicale citadine a connu des hauts et des bas, de même que certaines personnalités ont joué un rôle majeur dans son entretien et/ ou son renouveau. Des conclusions d'ordre plus général auraient aidé le lecteur à tirer quelques leçons sur les diverses attitudes sociales vis-à-vis de la pratique musicale, notamment amateure. Le rapport entre le désir des notables de faire rayonner la ville de Cork et les décisions de politique culturelle est passionnant. À ce sujet, l'étude sur les sociétés musicales - très majoritairement masculines avec trente-neuf sociétés contre six féminines - est parfaitement bien documentée et pourrait servir de modèle à d'autres recherches de ce type.

Grâce à l'imposant travail de Susan O'Regan, nous découvrons à quel point une ville moyenne, peu connue des musicologues en dehors des îles Britanniques, jouissait déjà par le passé d'une activité musicale riche et variée dont l'évolution interne se dessine ici avec une grande précision. Ainsi, par le biais du prisme étroit de la vie musicale, nous assistons à l'expansion même de Cork. Au-delà d'un inventaire exhaustif des manifestations musicales publiques dans cette cité

9. Norbert Elias, The Civilizing Process. Sociogenetic and Psychogenetic Investigations, édition revue, Oxford, Blackwell, 2000; Pierre Bourdieu, Distinction: A Social Critique of the Judgement of Taste, Cambridge, Harvard University Press, 1984. 
portuaire, deuxième ville d'Irlande en population avant que Belfast ne lui ravisse la place au milieu du XIX ${ }^{\mathrm{e}}$ siècle ${ }^{10}$, c'est le cœur même de Cork et de ses habitants qui bat sous nos yeux, telles les minutes de l'évolution démographique, économique, politique, sociale et culturelle d'une capitale régionale.

Yves Defrance

\section{Making Integral: Critical Essays on Richard Murphy, Benjamin Keatinge (dir.), Cork, Cork University Press, 2019, 362 p.}

En janvier 2018, l'un des derniers grands «Anglo-Irish poets», Richard Murphy, mourait à l'âge de 90 ans à son domicile près de Kandy, au Sri Lanka, l'île où il était né et avait passé son enfance, et où il était revenu mourir. Il y avait construit sa dernière œuvre (de pierre, et non de mots, car il créait aussi bien les deux), surnommée «l'Octogone», près de l'endroit où il avait habité enfant (une plantation de thé), du temps des beaux jours de l'Empire Britannique, quand son père, William Lindsay Murphy, était maire de Colombo à Ceylan, avant de devenir plus tard gouverneur général des Bahamas. Cet octogone sri lankais n'était pas le premier dans son genre, et faisait écho à une autre structure similaire construite sur une autre île par Murphy bien loin de là et bien longtemps auparavant, «l'Hexagone» irlandais sur Omey Island, dans le Connemara.

De l'ancienne Ceylan au Connemara, puis de retour à Ceylan, maintenant libérée du joug colonial et dénommée Sri Lanka, en passant par l'Angleterre, l'Amérique, l'Europe et l'Afrique du Sud: les pérégrinations géographiques variées de Richard Murphy, d'un bout à l'autre du globe, reflètent les incertitudes d'un poète toujours en quête d'un chez-lui, d'un home, sans jamais le trouver. Ces errances, d'un lieu à l'autre, traduisent aussi les angoisses d'un "Anglo-Irlandais » qui souffrit toute sa vie d'être situé dans ce tiret, dans cet espace intermédiaire entre deux pays, deux cultures, deux religions. Enfin, ces vagabondages masquent à moitié les ambiguïtés d'un homme constamment écartelé par sa bisexualité, dont de nombreux poèmes se font ouvertement l'écho.

Ce sont ces pérégrinations sans fin et cette perpétuelle quête d'une identité impossible qui caractérisent l'œuvre poétique de Richard Murphy, à laquelle Benjamin Keatinge ainsi que quinze autres éminents spécialistes de poésie irlandaise contemporaine se sont attelés dans ce bel ouvrage qu'est Making Integral: Critical Essays on Richard Murphy, paru en 2019 chez Cork University Press. Du très beau

10. Au début des années 1840 Cork comptait plus de 80000 habitants soit, à titre indicatif, près du double de la population rennaise à la même époque. Aujourd'hui Cork et Rennes ont une démographie équivalente avec à peu près 218000 habitants. Le développement de Belfast ne s'affirme, lui, qu'avec celui de l'industrialisation de ce port d'Ulster. 